\section{Prevalence of Dihydrofolate reductase gene mutations in Plasmodium falciparum isolate from pregnant women in Nigeria}

\author{
Olusola Ojurongbe,,12 Bukola D Tijani,, \\ Adegboyega A. Fawole, ${ }^{4}$ \\ Oluwaseyi A. Adeyeba, ${ }^{1}$ Juergen F. Kun ${ }^{2}$ \\ 'Department of Medical Microbiology and \\ Parasitology, Ladoke Akintola University \\ of Technology, Nigeria; ${ }^{2}$ Institute of \\ Tropical Medicine, University of \\ Tuebingen, Germany; ${ }^{3}$ Department of \\ Medical Microbiology and Parasitology, \\ University of Ilorin Teaching Hospital; \\ ${ }^{4}$ Department of Obstetrics and \\ Gynecology, University of Ilorin Teaching \\ Hospital, Nigeria
}

\section{Abstract}

We assessed the prevalence of Plasmodium falciparum and the frequency of the dhfr triple mutation that is associated with antifolate drug resistance among $P$. falciparum isolates obtained from pregnant women in Ilorin, Nigeria. The study included 179 women in the second and third trimester of pregnancy who have been exposed to intermittent preventive treatment in pregnancy (IPTp) with sulfadoxinepyrimethamine. Thick and thin blood films and PCR were used for malaria parasite detection. Blood group and hemoglobin concentration were also determined. Mutations in P. falciparum $\mathrm{dhfr}$ were analyzed by sequencing DNA obtained from blood spots on filter paper. Prevalence of $P$. falciparum in the population (PCR corrected) was $44.1 \%$ (79/179) with $66.7 \%$ and $33.3 \%$ in the second and third trimester, respectively. Primigravide (51.3\%) were more infected than multigravide (48.7\%) but the difference was not statistically significant. Women in blood group A had the highest $P$. falciparum malaria infection (30.8\%). The mean hemoglobin concentration was lower among those infected with malaria parasite. Also, more women with the malaria parasite (38.4\%) had anemia compare to those without (21.4\%). The prevalence of the $P$. falciparum dhfr mutant alleles was $64.1 \%, 61.5 \%, 38.5 \%$, and $12.8 \%$ for I51, R59, N108 and T108, respectively. None of the samples had the L164 mutation. The combined triple dhfr mutation $(51+59+108)$ in the population was $17.9 \%$ (7 of 39). Also, the prevalence of the triple mutant alleles was not significantly associated to the number of doses of SP taken by the women. These findings highlight the need for a regular assessment of IPTp/SP efficacy, and evaluation of possible alternative drugs.

\section{Introduction}

In all endemic areas the risk, frequency and severity of malaria infection are greater in pregnant women than in the same women before pregnancy or in their non-pregnant counterparts. Regardless of the pre-pregnancy level of immunity against malaria, clinical consequences of pregnancy-associated malaria include maternal anemia, low newborn birth weight (LBW), pre-term delivery, and increased prenatal morbidity. ${ }^{1}$

Prevention of malaria in pregnancy remains a major public health challenge and a priority for the Roll Back Malaria (RBM) Partnership. Methods of malaria prevention in pregnancy changed from a weekly or bimonthly chemoprophylaxis to intermittent preventive treatment (IPTp) and insecticide-treated bed nets (ITNs) at the beginning of the $21^{\text {st }}$ centrury. ${ }^{2}$ Sulfadoxine-pyrimethamine (SP) is the drug currently recommended for IPTp strategy. ${ }^{2}$ It has a good safety profile and remains a good option in endemic areas of Africa. ${ }^{3}$ IPTp-SP is used to treat pregnant women, regardless of their malaria infection status, with spaced therapeutic doses (2-3 doses) during the second and third trimesters of pregnancy.

Nigeria adopted the IPTp strategy in 2005 and the current National Malaria Treatment Guideline and Policy in Nigeria recommends SP as the drug of choice for IPTp and quinine for treatment of clinical malaria in all trimesters. Artemisinin based combination therapy (ACT) is considered a safe second-line agent in the second and third trimesters and may be used in the first trimester in cases in which there are no suitable alternatives. ${ }^{5}$ IPTpSP has been shown to reduce the risk of maternal anemia, placental parasitemia and low birth weight. ${ }^{6,7}$ The ongoing use of SP, either in treatment of uncomplicated malaria or as a method of malaria control by intermittent preventive treatment, makes it important to constantly study SP resistance and the way resistance spreads in populations. The resistance against the pyrimethamine component of the SP combination occurs due to multiple key mutations in the dihydrofolate reductase (dhfr) gene of the parasite. The first mutation to appear is serine to asparagine at codon 108 of the dhfr (S108N), followed by asparagine to isoleucine at codon 51 (N51I) and cysteine to arginine at codon 59 (C59R), leading to triple mutation-108N $+51 \mathrm{I}+59 \mathrm{R} .{ }^{8}$ The mutations in the dihydropteroate synthase (dhps) gene at codons 436, 437, 540 and 581 are associated with resistance to sulfadoxine..$^{8,9}$ Additional mutation at the dhfr codon 164, which is isoleucine to leucine (I164L), provides highlevel resistance against pyrimethamine, mostly found in the parasites in Southeast Asia ${ }^{10}$ and Latin America. ${ }^{11}$ However, few studies
Correspondence: Olusola Ojurongbe, Department of Medical Microbiology and Parasitology, Ladoke Akintola University of Technology, P.M.B 4400, Osogbo, Nigeria.

Key words: malaria, drug resistance, pregnancy, dhfr.

Conflict of interest: the authors have no conflict of interests.

Acknowledgment: the authors are grateful to all the women who volunteered to participate in the study.

Received for publication: 12 August 2011. Revision received: 5 October 2011.

Accepted for publication: 30 October 2011.

This work is licensed under a Creative Commons Attribution NonCommercial 3.0 License (CC BYNC 3.0).

(C) Copyright O. Ojurongbe et al., 2011

Licensee PAGEPress, Italy

Infectious Disease Reports 2011; 3:e16

doi:10.4081/idr.2011.e16

report the emergence of the $1164 \mathrm{~L}$ mutation in East Africa. ${ }^{12,13}$ The main aim of the present study, therefore, is to assess the prevalence of dhfr mutation in asymptomatic and submicroscopic $P$. falciparum isolates from pregnant women in Nigeria and to explore the possible association of such infection.

\section{Materials and Methods}

\section{Study area}

The study was carried out in Ilorin, the capital city of Kwara State, located in the middle belt region of Nigeria (lat. $830^{\circ} \mathrm{N}$, long. $430^{\circ} \mathrm{E}$ ) and has a Guinea Savannah type of vegetation. Average daily temperature is $37^{\circ} \mathrm{C}$, with a minimum mean temperature of $20^{\circ} \mathrm{C}$ and maximum mean temperature of $39^{\circ} \mathrm{C}$. Average rainfall is about $1,270 \mathrm{~mm}$ which falls almost entirely during the wet season from April to October. Patient recruitment took place at the maternity unit of the University of Ilorin Teaching Hospital (UITH), a tertiary health institution that functions as a teaching hospital.

\section{Study population and recruitment}

Pregnant women attending the antenatal clinic participated in the study. Apparently healthy pregnant women were adequately briefed about the purpose and benefits of this study and their consent was obtained before recruitment. Approval for the study was obtained from the UITH ethical committee. 


\section{Sample collection and processing}

Finger prick blood sample was collected from each subject for thick and thin blood smears, which were Giemsa stained and checked for $P$. falciparum parasites. The level of parasitemia (parasites/mL blood) was estimated from the results of a leukocyte count and a count of malarial parasites against 200 leukocytes in the thick smear. Two drops of blood were also blotted onto 3 MM Whatman filter paper for molecular studies. All subjects found to be infected were referred to a physician and treated with appropriate artemisinin combination therapy.

Hemoglobin estimation was carried out using photoelectric colorimeter according to Sahli's method, as previously described. ${ }^{14}$ Severe anemia was indicated by an $\mathrm{Hb}$ concentration of $7.0 \mathrm{~g} / \mathrm{dL}$ and mild-moderate anemia by an $\mathrm{Hb}$ concentration of 7.0-10.5 g/dL The $\mathrm{Hb}$ concentration used as the threshold for anemia was thus reduced from the usual $11.0 \mathrm{~g} / \mathrm{dL}$ to $10.5 \mathrm{~g} / \mathrm{dL}$, to allow for the hemodilution that occurs during pregnancy. ${ }^{15}$ The cellulose acetate membrane (CAM) was used to determine the genotype of each patient. ${ }^{16}$

\section{Detection of sub-microscopic malaria by PCR}

DNA was extracted from the dried blood spots on filter paper using the QIAamp ${ }^{\circledR D N A}$ Mini Kit (Qiagen, Hilden, Germany) following the manufacturer's protocol, and stored at $20^{\circ} \mathrm{C}$ until further analysis. Stevor gene was used for the detection of submicroscopic malaria as previously described. ${ }^{17}$ This gene was chosen because it is a multi-copied gene and we have previously reported that it has a $100 \%$ sensitivity and specificity. ${ }^{17}$

\section{Detection of DHFR gene mutation}

The PCR assays for dhfr mutation included a primary and semi-nested reaction to enhance specificity and sequencing was performed to determine the mutations. In the first reaction, $5.0 \mu \mathrm{L}$ of DNA extract were amplified in a final volume of $25 \mu \mathrm{L}$ containing $2.5 \mu \mathrm{L}$ of reaction buffer, 25mM dNTPs, 0.75 units of Taq DNA polymerase, and $0.5 \mu \mathrm{M}$ of forward and reverse primers. In the first reaction, a 654-basepair (bp) portion of the dhfr gene was amplified by primers DhfrFwd (5-TTTATATTTTCTCCTTTTTAT-3) and DhfrRvd (5-ACGCTAACAGA AATAATTTGA TACTC-3). For the second reaction, $3 \mu \mathrm{l}$ of amplified product from the first PCR product was added to the second PCR mixture. Dhfr Fwd2 (5-TAAGGTTGAAAGC AAAAATGAGG-3) and DhfrRvd were used to amplify a 581-bp fragment containing codons 51, 59, 108 and 164 . The primers were designed using the Vector NTI Advance $^{\mathrm{TM}}$ version 11.0. PCR products were subjected to electrophoresis on $1.5 \%$ agarose gels and visualized by transillumina- tion with ultraviolet light after staining with $\mathrm{SYBR}^{\circledR}$ Green. Amplicon sequence was determined using an ABI PRISIM 3100 Genetic Analyser (Applied Biosystem). Sequence results were analyzed using the Vector NTI Advance $^{\mathrm{TM}}$ version 11.0 software.

\section{Statistical analysis}

Statistical analysis was carried out using Graph-pad Instat (Graphpad Software Inc., San Diego, USA). Frequencies were compared using Fisher's exact tests and two sided $\mathrm{P}<0.05$ were considered statistically significant.

\section{Results}

A total of 179 pregnant women participated in the study. Their characteristics are shown in Table 1. The overall prevalence of women with $P$. falciparum parasitemia determined by PCR was 41.1\% (79/179). Gestational age, parity and blood group did not produce any significant effect on the presence of malaria parasitemia in the study population. Similarly, there was no significant difference between the hemoglobin concentrations of those with or without malaria. Anemia was more common among women with malaria parasitemia but the difference was not statistically significant (Table 1).

Out of the 79 positive cases obtained by stevor PCR, 39 isolates were successfully genotyped for the 3 point mutations on the dhfr gene. The frequency of the point mutations and the combination of the mutations are shown in Table 2. The prevalence of the mutant 108 alleles was $38.5 \%$ and $12.8 \%$ for N108 and T108, respectively. For the positions 51 and 59 , the prevalence of mutant alleles was $64.1 \%$ (I51) and $61.5 \%$ (R59). The prevalence of the triple mutant alleles (N108 + I51 + R59) in the population was $17.9 \%$. The prevalence of the triple mutant alleles with respect to number of doses of SP is compared in Table 3. No statistically significant difference was observed between the number of doses and the development of dhfr triple mutation.

\section{Discussion}

This study shows the prevalence of the dhfr mutations among parasites obtained from pregnant women with asymptomatic malaria after single or double dose(s) of SP used for IPTp in Nigeria. Dhfr resistant alleles have not yet been well correlated to SP effectiveness in pregnant women. We have previously reported a high prevalence of triple mutant dhfr alleles among children with uncomplicated falciparum malaria suggesting that there is a widespread pyrimethamine resistance in Nigeria. ${ }^{18}$ The high prevalence of triple mutant (17.9\%) and N108 mutation (38.5\%) of the dhfr alleles observed in asymptomatic $P$. falciparum cases may be an indication that SP has a limited duration of usefulness as IPTp in this part of the country. A change from wild-type S108 to N108 has been shown to be adequate to cause low-level pyrimethamine resistance both in vitro and in vivo. The addition of other mutations (C50 to R50, N51 to I51, and C59 to R59) yield a higher level of resistant. Also, more recently, the addition of a mutation at codon 164 (I164 to L164) has been shown to confer the highest level of resistance; although this mutation is not common in African isolates.

The emergence of SP-resistant parasites among pregnant women in Nigeria could be explained in part by previous pyrimethamine chemoprophylaxis usage. Weekly dose of pyrimethamine monotherapy was previously commonly used among pregnant women for prevention of malaria in pregnancy in Nigeria $^{19,20}$ and a recent survey (RL Opatokun,

Table 1. Characteristics of the pregnant women with and without submicroscopic $P$. falciparum infection in Ilorin, Nigeria.

\begin{tabular}{lllll} 
& Characteristics & $\begin{array}{l}\text { With malaria } \\
\text { parasite } \\
\mathbf{N}=79\end{array}$ & $\begin{array}{l}\text { Without malaria } \\
\text { parasite } \\
\mathbf{N}=100\end{array}$ & P \\
& Mean age \pm SD & $28.3 \pm 5.04$ & $29.1 \pm 5.16$ & 0.192 \\
Gestational age & $2^{\text {nd }}$ trimester & $43(54.4 \%)$ & $64(64.0 \%)$ & 0.195 \\
& $3^{\text {rd }}$ trimester & $36(45.6 \%)$ & $36(36.0 \%)$ & \\
\hline Parity & Primigravide & $36(46.6 \%)$ & $51(51.0 \%)$ & 0.470 \\
\multirow{5}{*}{ Blood group } & Multigravide & $43(54.4 \%)$ & $49(54.4 \%)$ & \\
& A & $27(34.2 \%)$ & $45(45 \%)$ & \\
& AB & $5(6.3 \%)$ & $2(2 \%)$ & 0.221 \\
& B & $14(17.7 \%)$ & $13(13 \%)$ & \\
& O+ & $32(40.5 \%)$ & $40(40 \%)$ & 0.347 \\
& O- & $1(1.3 \%)$ & $0(0.0 \%)$ & 0.354 \\
\hline
\end{tabular}


Table 2. Prevalence of dhfr mutations in P. falciparum isolates obtained from pregnant women in Ilorin, Nigeria.

\begin{tabular}{lllll} 
Gene & $\begin{array}{l}\text { Amino Acid } \\
\text { Wild/Mutant }\end{array}$ & $\begin{array}{l}\text { N }=39 \\
\text { Wild }\end{array}$ & $\begin{array}{l}\text { Mutant } \\
\text { Mixed }\end{array}$ \\
51 & $\mathrm{~N} / \mathrm{I}$ & $10(25.6 \%)$ & $25(64.1 \%)$ & $4(10.3 \%)$ \\
59 & $\mathrm{C} / \mathrm{R}$ & $13(33.3 \%)$ & $24(61.5 \%)$ & $2(5.1 \%)$ \\
\hline 108 & $\mathrm{~S} / \mathrm{N}$ & $19(48.7 \%)$ & $15(38.5 \%)$ & 0 \\
& $\mathrm{~S} / \mathrm{T}$ & & $5(12.8 \%)$ & \\
164 & $\mathrm{I} / \mathrm{H}$ & $40(100 \%)$ & 0 & 0 \\
\hline $51+59$ & & $13(33.3 \%)$ & $26(66.7 \%)$ & 0 \\
$51+108$ & & $3(7.7 \%)$ & $10(25.6 \%)$ & $26(66.7 \%)$ \\
\hline $59+108$ & & $1(2.6 \%)$ & $7(17.9 \%)$ & $31(79.5 \%)$ \\
$51+59+108$ & & $0(37.5 \%)$ & $7(17.9 \%)$ & $32(82.1 \%)$ \\
\hline
\end{tabular}

Table 3. The effect of number of doses of SP on the presence of triple dhfr mutation.

\begin{tabular}{llll} 
SP Dose & N. & Dhfir triple mutation & P \\
1 dose & 25 & $5(20 \%)$ & \\
$>1$ dose & 14 & $2(14 \%)$ & 1.0000 \\
\hline Total & 39 & $7(17.9 \%)$ & \\
\hline
\end{tabular}

unpublished data, 2011) in Osogbo, South Western Nigeria the country showed that over $10 \%$ of pregnant women are still using Daraprim ${ }^{\circledR}$, a weekly pyrimethamine monotherepy for malaria prevention. Current IPT-Sp treatment and previous pyrimethamine monotherapy in pregnant women could have caused bias by selecting resistant parasites on an individual basis and successively over time. An earlier study in Tanzania showed that pyrimethamine chemoprophylaxis in children was paralleled by a rapid emergence and worsening of drug resistance, arguing against the use of pyrimethamine as a monosubstance. ${ }^{8}$ In this study, we did not observe a significant difference in the prevalence of triple dhfr mutant alleles between women that took a single dose compared to those who took 2 or 3 doses. One limitation of this study was that the drugs were not taken under the supervision of the physician. The women were given the drugs freely to take home and the compliance of the women cannot be independently verified.

The prevalence of asymptomatic malaria in our study area is $44 \%$. Variable prevalence of malaria ranging from low $(7.7 \%),{ }^{21}$ to high $(75 \%)^{22}$ has been reported among pregnant women in Nigeria. Agomo et al. ${ }^{21}$ attributed these differences in the reported prevalence rates to the skill and experience of the laboratory personnel involved in blood film preparation, staining, and reading of the slides. In our study, we relied on PCR for the true prevalence of asymptomatic malaria in the study population. PCR-based assays remain the most sensitive and specific methods for the detection of malaria parasites. ${ }^{17}$

Although anemia was more common among pregnant women who had malaria parasite compared to those who did not, the difference was not significant. One explanation for this observation could be because most of the malaria positive pregnant women had low density and submicroscopic parasitemia. Thus, these parasites are unlikely to have contributed significantly to the presence of anemia in this study population.

In conclusion, we report a high prevalence of dhfr gene mutations in submicroscopic falciparum parasite obtained from pregnant women in Nigeria. Replacement of SP by an alternative drug will be necessary in the near future for successful implementation of IPTp. Regular assessment of sulfadoxine/pyrimethamine and its correlation to molecular markers in pregnant women is also recommended.

\section{References}

1. Steketee RW, Wirima JJ, Campbell CC. Developing effective strategies for malaria prevention programs for pregnant African women. Am J Trop Med Hyg 1996;55:95100.

2. WHO. A strategic framework for malaria prevention and control during pregnancy in the African region. Brazzaville, Republic of Congo, 2004.

3. Mubyazi G, Bloch P, Kamugisha M, et al. Intermittent preventive treatment of malaria during pregnancy: a qualitative study of knowledge, attitudes and practices of district health managers, antenatal care staff and pregnant women in
Korogwe District, North-Eastern Tanzania. Malar J 2005;4:31.

4. Falade CO, Yusuf BO, Fadero FF, et al. Intermittent preventive treatment with sulphadoxine-pyrimethamine is effective in preventing maternal and placental malaria in Ibadan, south-western Nigeria. Malar J 2007;6:88.

5. FMOH. National guidelines and strategies for malaria prevention and control during pregnancy. A publication of the Federal Ministry of Health, Nigeria. Abuja Nigeria,

6. Aziken ME, Akubuo KK, Gharoro EP. Efficacy of intermittent preventive treatment with sulfadoxine-pyrimethamine on placental parasitemia in pregnant women in midwestern Nigeria. Int $\mathrm{J}$ Gynaecol Obstet 2011;112:30-3.

7. Oyibo WA, Agomo CO. Scaling up of intermittent preventive treatment of malaria in pregnancy using sulphadoxine-pyrimethamine: prospects and challenges. Matern Child Health J 2011;15:542-52.

8. Gregson A, Plowe CV. Mechanisms of resistance of malaria parasites to antifolates. Pharmacol Rev 2005;57:117-45.

9. Nzila AM, Mberu EK, Sulo J, Dayo H, et al. Towards an understanding of the mechanism of pyrimethamine-sulfadoxine resistance in Plasmodium falciparum: genotyping of dihydrofolate reductase and dihydropteroate synthase of Kenyan parasites. Antimicrob Agents Chemother 2000;44:991-6.

10. Biswas S, Escalante A, Chaiyaroj S, et al. Prevalence of point mutations in the dihydrofolate reductase and dihydropteroate synthetase genes of Plasmodium falciparum isolates from India and Thailand: a molecular epidemiologic study. Trop Med Int Health 2000;5:737-43.

11. Plowe CV, Cortese JF, Djimde A, et al. Mutations in Plasmodium falciparum dihydrofolate reductase and dihydropteroate synthase and epidemiologic patterns of pyrimethamine-sulfadoxine use and resistance. J Infect Dis 1997;176: 1590-6.

12. Alker AP, Mwapasa V, Purfield A, et al. Mutations associated with sulfadoxinepyrimethamine and chlorproguanil resistance in Plasmodium falciparum isolates from Blantyre, Malawi. Antimicrob Agents Chemother 2005;49:3919-21.

13. Kiara SM, Okombo J, Masseno V, et al. In vitro activity of antifolate and polymorphism in dihydrofolate reductase of Plasmodium falciparum isolates from the Kenyan coast: emergence of parasites with Ile-164-Leu mutation. Antimicrob Agents Chemother 2009;53:3793-8.

14. Barduagni P, Ahmed AS, Curtale F, et al. Performance of Sahli and colour scale methods in diagnosing anaemia among 
school children in low prevalence areas. Trop Med Int Health 2003;8:615-8.

15. Alusala DN, Estambale BB, Magnussen P, et al. Predictors of serum ferritin and haemoglobin during pregnancy, in a malaria-endemic area of western Kenya. Ann Trop Med Parasitol 2008;102:297-308.

16. Wild BJ, Bain BJ. Detection and quantitation of normal and variant haemoglobins: an analytical review. Ann Clin Biochem 2004;41:355-69.

17. Oyedeji SI, Awobode HO, Monday GC, et al. Comparison of PCR-based detection of Plasmodium falciparum infections based on single and multicopy genes. Malar $\mathrm{J}$ 2007;6:112.

18. Ojurongbe 0, Oyedeji SI, Oyibo WA, et al. Molecular surveillance of drug-resistant Plasmodium falciparum in two distinct geographical areas of Nigeria. Wien Klin Wochenschr 2010;122:681-5.

19. Fawole AO, Onyeaso NC. Perception and practice of malaria prophylaxis in pregnancy among primary health care providers in Ibadan, Nigeria. West Afr J Med 2008;27:92-6.

20. Yusuf OB, Dada-Adegbola HO, Ajayi IO, Falade CO. Malaria prevention practices among mothers delivering in an urban hospital in southwest Nigeria. J Vector Borne Dis 2008;45:217-24.

21. Agomo C0, Oyibo WA, Anorlu RI, Agomo PU. Prevalence of malaria in pregnant women in Lagos, South-West Nigeria. Korean J Parasitol 2009;47:179-83.

22. Adefioye OA, Adeyeba OA, Hassan WO, Oyeniran OA. Prevalence of malaria parasite infection among pregnant women in Osogbo, southwest, Nigeria. AmericanEurasian J Sci Res 2007;2:43-5. 